\title{
HOSPITALIZAÇÕES POR DIABETES MELLITUS EM ADULTOS E RELAÇÃO COM EXPANSÃO DA ATENÇÃO PRIMÁRIA NO PARANÁ
}

\section{Thaisnara Hassan Ribeiro}

Discente do curso de Medicina do Centro Universitário de Maringá - UniCesumar, Maringá - PR, Brasil.

\section{Camila Lauana Magri}

Discente do curso de Medicina do Centro Universitário de Maringá - UniCesumar, Maringá - PR, Brasil.

\section{Aliny de Lima Santos}

Doutora em Enfermagem. Docente nos cursos de Medicina e Enfermagem no Centro Universitário de Maringá - UniCesumar, Maringá - PR, Brasil.
RESUMO: Estudo com objetivo de analisar os coeficientes de hospitalização por diabetes mellitus no Estado do Paraná no período de 2005-2015 e contextualizar com estimativa da cobertura por equipes da Estratégia Saúde da Família no mesmo estado e período. Estudo ecológico, de séries temporais que analisou as internações entre 2005 e 2015 obtidas no banco de dados do Departamento de Informática do Sistema Único de Saúde. As taxas foram calculadas e analisadas segundo sexo e faixa etária. Houve quedas consideráveis em todas as faixas etárias estudadas e em ambos os sexos, com destaque para quedas mais expressivas nas mulheres, apresentando maior redução na variação percentual $(-43,8 \%)$ quando comparadas aos homens (-22,9\%). Constatou-se também que quanto maior a faixa etária, maiores os coeficientes de hospitalização.

PALAVRAS-CHAVE: Hospitalização; Avaliação em saúde; Diabetes Mellitus; Estratégia saúde da família.

\section{HOSPITALIZATION DUE TO DIABETES MELLITUS IN ADULTS AND ITS RELATIONSHIP WITH FIRST AID EXPANSION IN PARANÁ, BRAZIL}

\begin{abstract}
Hospitalization coefficients due to diabetes mellitus are analyzed for the state of Paraná, Brazil, between 2005 and 2015, and contextualized for coverage estimates by Family Health Strategy teams in the same state and during the same period. Current ecological and temporal study analyzes hospitalization between 2005 and 2015 retrieved from databases of the Information Department of the National Health System. Rates were calculated and analyzed according to gender and age. Significant decreases occurred in all age brackets and in gender rates, especially in females, with greatest percentage rate $(-43.8 \%)$ when compared to that of males $(-22.9 \%)$. Results show that the higher the age bracket, the greater are the hospitalization coefficient rates.
\end{abstract}

KEY WORDS: Hospitalization; Health evaluation; Diabetes Mellitus, Family health strategy.

\section{INTRODUÇÃO}

O Diabetes Mellitus (DM) é uma das doenças crônicas mais

Autor correspondente

Aliny Lima Santos

aliny.lima.santos@gmail.com prevalentes no Brasil, que em 2015 acometeu cerca de 14 milhões de brasileiros, deixando o país na quarta posição na relação entre os dez com maior número de casos no mundo ${ }^{1}$. Em 2014, a prevalência mundial do DM em adultos estava em cerca de 422 milhões, sendo que 
esse número era de 108 milhões em 1980, o que denota um incremento de quase quatro vezes o número de acometidos em menos de 35 anos. Além disso, o número de óbitos decorrentes do DM chegou a 1,5 milhão em $2012^{2}$.

Juntamente com o crescimento da prevalência e incidência da obesidade e das dislipidemias, resultantes do estilo de vida da sociedade moderna, as estimativas globais para o DM são deveras preocupantes. Um estudo publicado pela Internacional Diabetes Federation, em dezembro de 2015, demonstrou que a perspectiva de casos de DM para 2040 no mundo, é de aproximadamente 328,4 milhões, para o sexo masculino, e 313,3 milhões para o sexo feminino ${ }^{3}$. Assim como doenças respiratórias, cardiovasculares e os cânceres, o DM pertence ao grupo das principais Doenças Crônicas Não Transmissíveis (DCNT), responsáveis por $51,6 \%$ do total de óbitos na população de 30 a 69 anos no Brasil em $2015^{4}$.

Em perspectiva nacional, estudo realizado em todas as capitais, em 2017, evidenciou que, no conjunto das 27 cidades, a prevalência do diagnóstico médico prévio de DM em pessoas com mais de 18 anos foi de 7,6\%, sendo que em ambos os sexos, este diagnóstico se tornou mais comum com o avanço da idade e em indivíduos com até oito anos de estudo5. Ainda nesse estudo, observouse prevalência do diagnóstico no sexo feminino $(8,1 \%)$ em detrimento do masculino $(7,1 \%)$ independente da idade ou da cidade investigada. Tal resultado ressalta a interferência da idade e escolaridade no acometimento pela doença 5 .

$\mathrm{Na}$ atual realidade do país, uma combinação de fatores relacionados com a organização dos serviços de saúde, de modo a favorecerem o acesso ao diagnóstico precoce, bem como os comportamentos individuais dos sujeitos, o início insidioso dos sintomas, entre tantos outros, contribui para que a doença permaneça não detectada por anos, favorecendo o desenvolvimento de complicações. Estas, por sua vez, são ainda mais agravantes em países em desenvolvimento, que contam com $83,8 \%$ de todos os casos de DM não diagnosticados ${ }^{1}$.

O DM destaca-se por ser uma doença de alta morbimortalidade, comprometendo a qualidade de vida do paciente e demandando alto custo para realização do controle e tratamento de complicações. Estas podem ser divididas em agudas (hipoglicemia, hiperglicemia e cetoacidose diabética) e crônicas (retinopatia, nefropatia, doença cerebrovascular e doença vascular periférica). O aparecimento de complicações se agrava em pessoas que não realizam atividades de autocuidado, tais como práticas de autocuidado e automanejo da doença e uso adequado das medicações necessárias ${ }^{6}$.

Ademais, sabe-se que o DM se associa a condições metabólicas diversas, como as dislipidemias, havendo concordância de até $53,4 \%$ entre as duas doenças, justificada pela influência do DM no metabolismo dos lipídeos, além do impacto do estilo de vida, sedentarismo e fatores sociodemográficos, que também colaboram para o desenvolvimento de alterações do perfil lipídico do paciente ${ }^{7}$.

Por conseguinte, o DM invariavelmente acarreta alta taxa de hospitalizações e maior utilização dos serviços de saúde por parte do paciente, onerando o sistema de saúde mundial, porém, de forma mais impactante, os países em desenvolvimento ${ }^{1}$, os quais possuem grandes dificuldades na implantação de serviços de saúde modernos e eficientes.

As complicações relacionadas ao DM previamente citadas requerem intervenções, ressaltando-se ainda que o DM constitui uma Condição Sensível à Atenção Primária (CSAP). Há evidências de que a atuação adequada de profissionais e gestores de saúde em nível da Atenção Primária à Saúde (APS) pode colaborar para a redução do número de hospitalizações e óbitos pela doença, principalmente por ações das equipes da Estratégia Saúde da Família (ESF) ${ }^{8}$. Considera-se, ainda, que este impacto deve-se à efetivação de programas do Ministério da Saúde, como o Programa Nacional de Hipertensão e Diabetes Mellitus - (HiperDia), criado como forma de qualificar a assistência aos portadores da doença por meio da garantia da terapia medicamentosa, monitoramento das condições clínicas e, por conseguinte, prevenção das complicações a ela relacionadas ${ }^{4}$.

No Brasil, a APS é implementada por meio da ESF, que reflete o conjunto de ações e serviços aos quais a população tem acesso. Dessa forma, um aumento da cobertura populacional da ESF deveria fortalecer a diminuição da morbimortalidade de doenças sensíveis à atenção primária, como o $\mathrm{DM}^{8}$.

Deste modo, a relevância do DM no cenário 
epidemiológico nacional na atualidade justifica a investigação e a interpretação dos coeficientes de internação por essa doença. A análise da tendência desses coeficientes com o passar do tempo pode favorecer a gestão no controle, monitoramento e desenvolvimento de ações para prevenção da morbimortalidade causada pelo diabetes ${ }^{8}$.

Ademais, o índice de hospitalizações por DM pode ainda ser utilizado para uma análise da qualidade do sistema de atenção em nível primário, uma vez que este é responsável pelo cuidado e acompanhamento desses pacientes. As evidências mostram que a melhoria da qualidade e a maior abrangência do serviço ambulatorial resultam na redução nos números de hospitalizações por motivos crônicos?

O estudo proposto se valoriza por buscar uma análise dos índices de internações causadas por complicações advindas do DM no Estado do Paraná, durante o período de 2005-2015. Trata-se de uma análise indireta utilizando um panorama geral da qualidade e da efetividade da abrangência dos serviços de saúde prestados em nível primário. Deste modo, o estudo teve como objetivo analisar os coeficientes de hospitalização por DM no Estado do Paraná no período de 2005-2015 e contextualizar com estimativa da cobertura por equipes da Estratégia Saúde da Família no mesmo Estado e período.

\section{METODOLOGIA}

Estudo de série temporal, do tipo ecológico, com abordagem quantitativa. Utilizaram-se informações referentes às internações hospitalares nos anos de 2005 a 2015 obtidas exclusivamente no banco de dados do Departamento de Informática do SUS (DATASUS)/ Ministério da Saúde na seção "Informações de Saúde". Acessou-se o item informações "Epidemiológicas e Morbidade", nas opções que correspondem "Morbidade hospitalar do SUS ("Geral, por local de internação - 1984 a 2007"/"Geral, por local de internação - a partir de $2008 ")$.

Por sua vez, os dados relacionados à cobertura populacional pela ESF foram coletados a partir do sítio eletrônico do Departamento da Atenção Básica (DAB) do Ministério da Saúde. Tendo em vista que o sistema emite a cobertura para cada mês do ano, calcularam-se as coberturas populacionais estimadas da ESF para o Estado do Paraná, adotando-se como base a população coberta pela ESF apenas nos meses de dezembro de cada ano da série histórica; e número de residentes no Estado para cada respectivo ano, calculando assim o coeficiente de cobertura da ESF no Estado do Paraná.

As taxas de internações hospitalares foram calculadas segundo sexo e faixa etária, dividindo-se o número total de internações por DM de cada ano, pela população do mesmo período ${ }^{10}$, sexo e faixa etária, segundo e?? multiplicado por 100.000 . Vale salientar que os coeficientes foram padronizados segundo população do Paraná referente ao ano de 2012. Foi também feito o cálculo da variação das taxas de hospitalização considerando o primeiro e o último coeficiente de hospitalização verificados, dentro do período estudado, segundo sexo e faixa etária, apresentado em valor percentual ((V2-V1) $N 1 * 100)$.

Os cálculos dos coeficientes de hospitalizações de acordo com as séries históricas foram elaborados em planilhas do Microsoft Excel ${ }^{\circledR}$ e analisados segundo estatística descritiva simples, por meio de análise de variação percentual e cálculo de médias, apresentados em tabelas e gráficos.

O estudo, de acordo com a resolução $\mathrm{N}^{\circ} 466$, de 12 de dezembro de 2012, do Conselho Nacional de Saúde, não apresenta implicações éticas, por utilizar dados secundários, nos quais não constarão informações que possam identificar os indivíduos. Deste modo obteve-se a liberação do Comitê de Ética em Pesquisa do Comitê de Ética em Pesquisa do Centro Universitário de Maringá (UniCesumar) com parecer n $n^{0}$ 1.673.265 (CAAE: 58326716.7.0000.5539), emitido em agosto de 2016.

\section{RESULTADOS}

Fazendo uma análise do número de hospitalizações por DM de residentes do Paraná entre os anos de 2005 e 2015 é possível observar que, de maneira geral, essas taxas diminuíram em todas as faixas etárias e em ambos os sexos durante o período analisado, como demonstrado nas variações percentuais (Tabela 1). 
Tabela 1. Distribuição dos coeficientes de hospitalização por DM (por 100.000 habitantes) em residentes no Paraná, segundo sexo e faixa etária, no período de 2005 a 2015. Maringá, Paraná, 2016

\begin{tabular}{ccccccccccc}
\hline \multirow{2}{*}{ Ano } & \multicolumn{2}{c}{ 20-29 } & \multicolumn{2}{c}{$30-39$} & \multicolumn{2}{c}{ 40-49 } & \multicolumn{2}{c}{$50-59$} & \multicolumn{2}{c}{ Total } \\
\cline { 2 - 10 } & Fem* & Masc** & Fem & Masc & Fem & Masc & Fem & Masc & Fem & Masc \\
\hline 2005 & 22,9 & 14,5 & 32,6 & 26,4 & 79,1 & 58,3 & 242,6 & 166,4 & 377,2 & 265,6 \\
2006 & 21,1 & 15,4 & 26,7 & 24,8 & 77,7 & 57,2 & 217,0 & 151,9 & 342,5 & 249,3 \\
2007 & 23,7 & 11,3 & 29,1 & 26,0 & 65,1 & 53,4 & 200,3 & 127,3 & 318,2 & 218,0 \\
2008 & 22,9 & 16,6 & 37,0 & 30,2 & 75,0 & 61,7 & 217,1 & 140,8 & 352,0 & 249,3 \\
2009 & 19,3 & 13,9 & 29,2 & 25,3 & 84,2 & 68,4 & 227,1 & 147,8 & 359,8 & 255,4 \\
2010 & 19,8 & 17,5 & 36,8 & 29,1 & 76,9 & 69,1 & 223,8 & 163,1 & 357,3 & 278,8 \\
2011 & 18,9 & 15,6 & 30,0 & 28,2 & 67,1 & 59,6 & 192,2 & 142,4 & 308,2 & 245,8 \\
2012 & 17,3 & 13,5 & 29,5 & 24,5 & 67,2 & 57,7 & 205,0 & 145,2 & 319,0 & 240,9 \\
2013 & 17,4 & 12,5 & 27,8 & 26,9 & 68,8 & 63,8 & 169,6 & 146,7 & 283,6 & 249,9 \\
2014 & 20,1 & 16,8 & 27,2 & 21,0 & 63,4 & 59,1 & 142,0 & 130,3 & 252,7 & 227,2 \\
2015 & 19,6 & 14,3 & 24,5 & 22,7 & 48,1 & 50,3 & 119,6 & 117,5 & 211,8 & 204,8 \\
Var. \%*** & $-14,4$ & $-1,3$ & $-24,8$ & $-14,0$ & $-39,2$ & $-13,7$ & $-50,7$ & $-29,4$ & $-43,8$ & $-22,9$ \\
\hline
\end{tabular}

Dados obtidos no Datasus. *Sexo Feminino; **Sexo Masculino, ***Variação percentual entre os períodos estudados ((2015 - 2005)/ $2005 * 100)$.

Analisando individualmente as faixas etárias em estudo na Figura 1, observa-se que a queda das hospitalizações é mais expressiva nas mulheres, ainda que estas apresentem os maiores coeficientes durante todo o período e todas as faixas etárias estudadas. Mediante o conjunto de dados, é também possível confirmar o aumento dos coeficientes de acordo com o aumento das faixas etárias.

Observa-se, na Figura 1, que quanto maior a faixa etária, maior a discrepância entre os coeficientes verificados em homens e mulheres. Além disso, as variações das duas primeiras faixas etárias são discretas, apresentando um comportamento quase linear, característica esta que se modifica com o avanço da idade, uma vez que quanto maior a faixa etária, maiores as oscilações dos coeficientes.

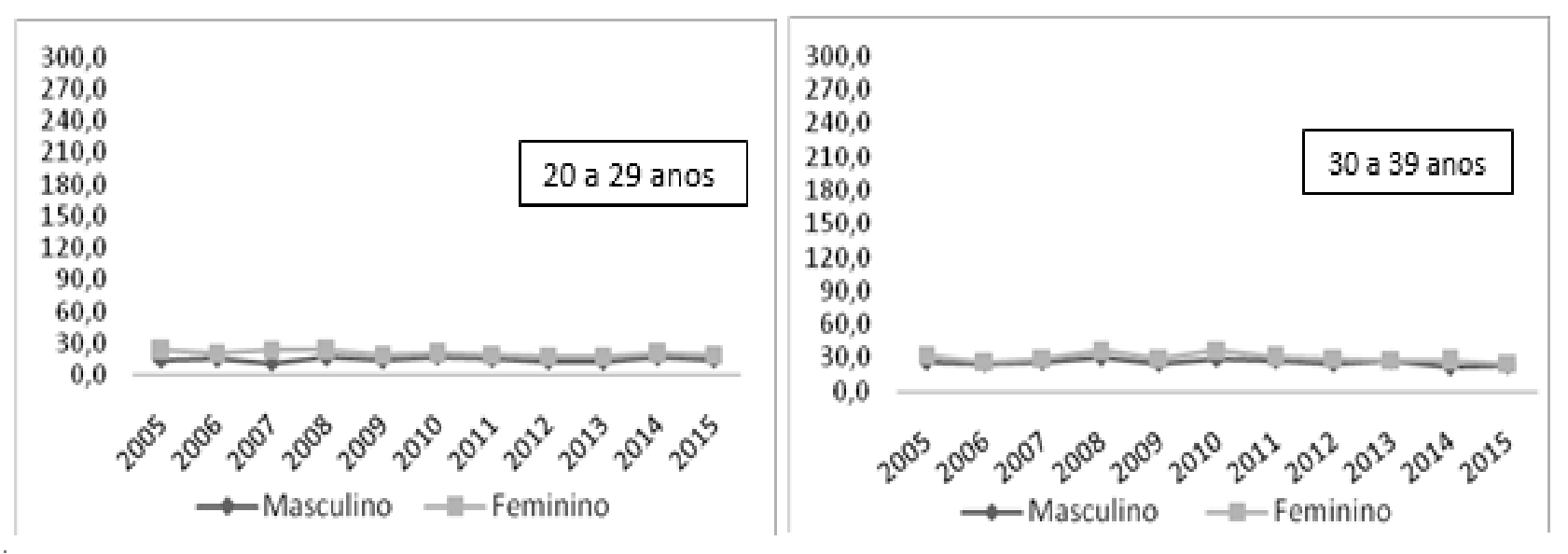




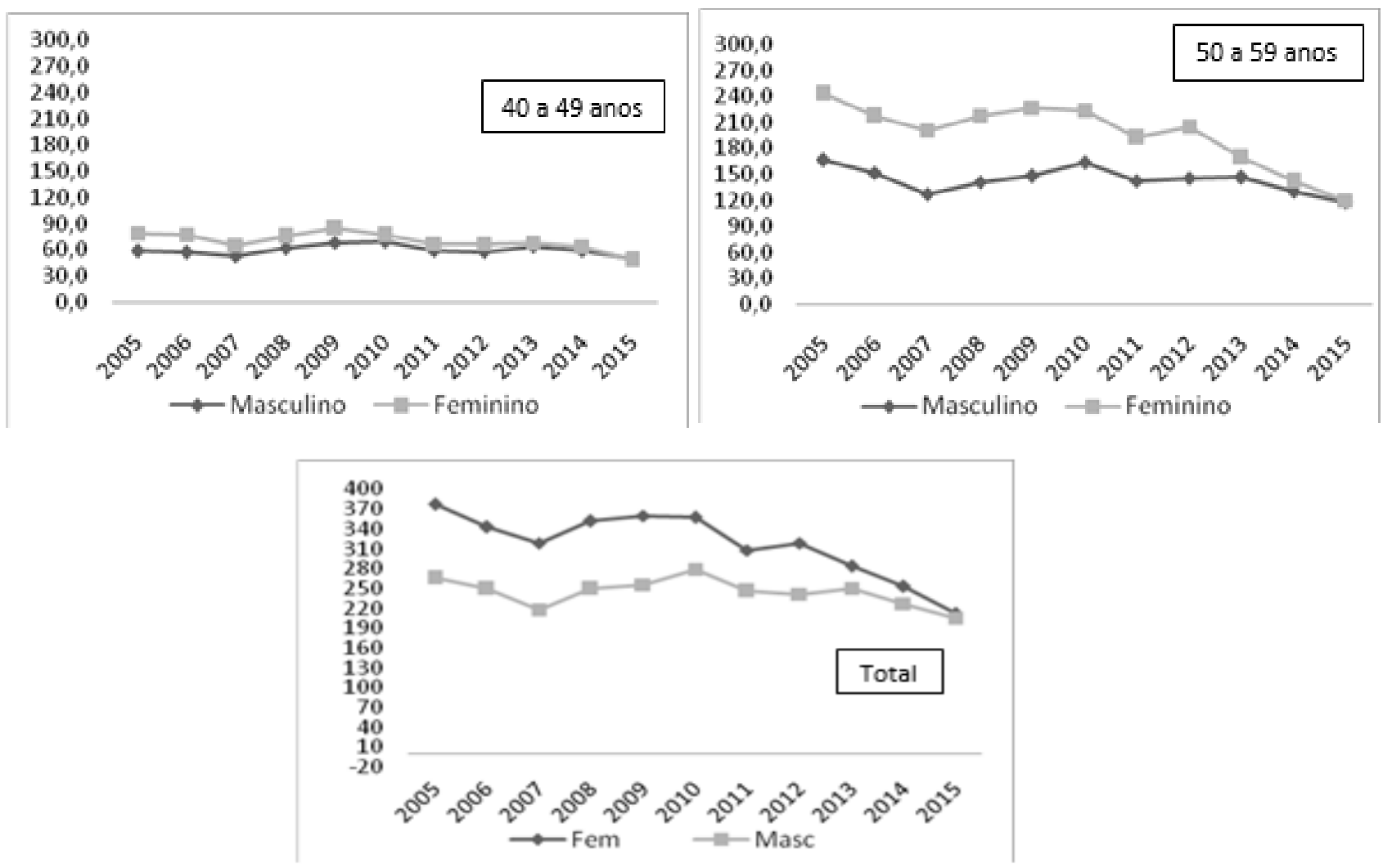

Figura 1. Coeficientes de hospitalização por DM (por 100.000 habitantes) em residentes do Paraná, no período de 2005 a 2015, segundo sexo e faixa etária. Maringá, PR, Brasil. 2016.

$\mathrm{Na}$ análise da cobertura da ESF no Estado, evidenciou-se aumento de 4.708 .215 pessoas cobertas pelo serviço em 2005 para 7.239.417 em 2015. Tal resultado imprime em aumento de $44,2 \%$ da proporção de cobertura populacional pela ESF, indo de $47,4 \%$ de população coberta em 2005 para 68,4\%, em 2015, como demonstrado na Figura 2.

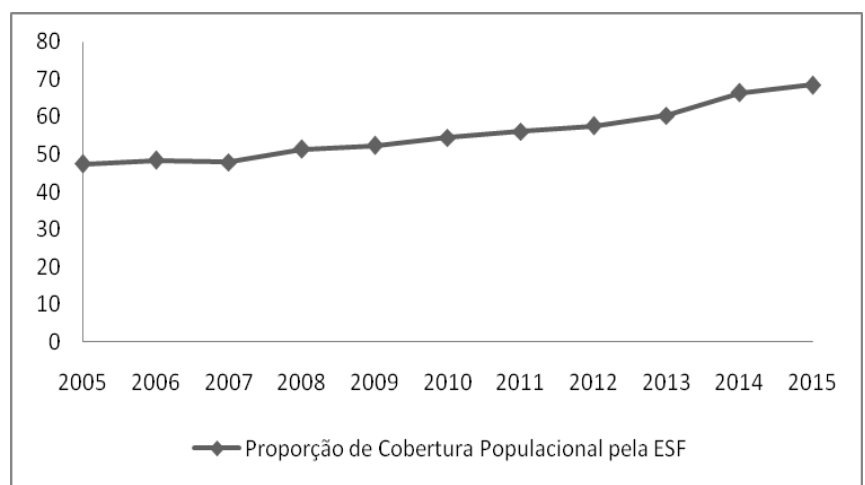

Figura 2. Proporção de cobertura populacional estimada pela ESF entre os anos de 2005 e 2015. Maringá, Paraná, Brasil, 2018.

\section{DISCUSSÃO}

O decréscimo no número de internações evidenciado nesse estudo pode ser atribuído a diversos fatores, dentre eles o aumento do percentual de cobertura assistencial da Estratégia Saúde da Família e por sua vez uma melhora no acesso aos serviços de saúde, indicadores que podem impactar na redução das internações evitáveis. Corroborando este fato, os achados do estudo evidenciam, para além da redução da taxa de hospitalização, o aumento de cobertura populacional estimada da ESF no período em estudo.

Nos últimos anos houve expansão da ESF em todo o território nacional ${ }^{1}$ com forte indução e apoio do Ministério da Saúde, sendo considerada, enquanto estratégia prioritária de estruturação da APS, e principal porta de entrada do SUS. A ESF tem papel fundamental no primeiro contato, na longitudinalidade e na coordenação do cuidado, devendo operar como base de estruturação das redes de atenção, com suporte dos serviços de apoio 
diagnóstico, assistência especializada e hospitalar ${ }^{11}$.

Não obstante, deve ainda ser considerado como um fator importante na queda das hospitalizações por DM as melhorias na organização do serviço ambulatorial, a composição das equipes de saúde, a qualificação dos profissionais e o estabelecimento de vínculos trabalhistas, dentre outros elementos relacionados à dimensão estrutural ${ }^{12}$. Ou seja, houve melhorias nas condições dos prestadores de serviço de saúde, o que por consequência pode ter impactado na melhoria dos serviços prestados e na saúde da população que usufrui desses serviços, influenciando possivelmente a diminuição das internações por complicações do DM no Paraná ${ }^{14}$.

Mesmo que a implementação da ESF não seja ainda totalmente eficaz na prevenção de complicações relacionadas ao DM, a melhoria do acesso ao serviço de saúde e melhor capacidade do sistema em diagnosticar e alimentar as bases de dados com informações necessárias tem relação com a diminuição dos casos de internação pela doença em questão ${ }^{14}$.

Essa relação está explicitada na diminuição dessas internações à medida que a cobertura pela ESF aumentou nos anos analisados, com maior demanda de tempo de cuidado e maior dependência dos hábitos de vida e perfil de morbidade dos pacientes, fatores que podem dificultar a avaliação do impacto da ESF sobre tal condição $0^{15}$.

No que diz respeito ao sexo feminino, o presente estudo demonstrou que houve declínio expressivo nas taxas de internações hospitalares, com velocidade de queda maior com o avançar da idade, principalmente as faixas etárias de 30 a 59 anos. No sexo masculino, por sua vez, foi observado crescimento nos coeficientes de hospitalização referentes apenas as maiores faixas etárias, sendo a de 50 a 59 anos ou mais, com declínio ou estabilidade nas demais faixas. Apesar do DM atingir grupos etários diversos, o que corrobora a mudança do perfil epidemiológico da doença observado na atualidade, em que faixas etárias mais longevas apresentam taxas mais elevadas de hospitalização, característica do perfil crônico e evolutivo da doença ${ }^{16}$.

Quando analisadas separadamente por sexo, constata-se que as taxas para as mulheres se mantiveram superiores em todo o período estudado, o que ao encontro dos achados de outro estudo abordando a hospitalização por $\mathrm{DM}^{17}$. A predominância das hospitalizações femininas pode ser vista como reflexo da maior prevalência da doença neste $\operatorname{sexo}^{18}$.

Embora tenham apresentado maiores coeficientes no período, as hospitalizações em mulheres sofreram queda durante o período, fato que pode estar associado à demanda dos serviços de saúde na assistência ambulatorial, composta majoritariamente por programas que beneficiam o cuidado à saúde da mulher nos diferentes ciclos da vida, e que acabam por favorecer e repercutir na maior procura e utilização de serviços de saúde, especialmente por mulheres com mais idade ${ }^{19}$.

Dessa forma, acredita-se que haja o predomínio das mulheres na utilização de serviços de saúde, o que corrobora o resultado de um ensaio clínico randomizado realizado, cujas mulheres apresentaram 1.4 vezes mais chance de utilizarem o serviço de saúde quando comparadas com os homens ${ }^{20}$.

Por outro lado, a tendência crescente nas hospitalizações em homens pode estar associada à sua reduzida procura pelos serviços de saúde, à resistência em realizar o autocuidado, associada à negligência diante das ações preventivas, principalmente as que estão voltadas para as doenças de caráter crônico degenerativo, buscando o serviço de saúde muitas vezes quando a hospitalização já se faz necessária, ou seja, em estágios mais avançados da doença ${ }^{21}$.

De modo mais específico, um estudo sobre o comportamento de autocuidado em homens com DM mostrou que a maioria deles desconhecia os sintomas de descompensação e de complicações da doença, demonstrando, ainda, ausência de adesão adequada ao tratamento e de acompanhamento frequente dos níveis glicêmicos, além de índice de massa corporal, relação cintura-quadril e glicemia com médias acima do preconizado em indivíduos saudáveis ${ }^{22}$.

Desta forma, quando os homens vivenciam alterações mais graves no estado de saúde, acabam procurando a assistência hospitalar, e assim, a sua entrada nos serviços de saúde acaba ocorrendo na alta complexidade, observado pelo aumento das taxas de internações no sexo masculino ${ }^{22}$. 
Sugere-se, então, que a utilização dos serviços de saúde no âmbito da assistência ambulatorial configura-se como agente fundamental para o diagnóstico precoce da doença, melhor controle glicêmico, acompanhamento de possíveis complicações e, consequentemente, prevenção de agravos e hospitalizações ${ }^{23}$.

Sendo assim, o decréscimo nas internações por DM no Paraná pode estar relacionado com o melhor acesso e a qualidade do cuidado na APS de forma oportuna e eficaz, o que leva à melhoria dos cuidados preventivos e consequente redução da doença. Desta maneira, considerando o aumento da população acompanhada e tratada pela APS, as taxas de internações por DM tendem a decrescer.

\section{CONCLUSÃO}

O estudo mostrou, de forma geral, queda nos coeficientes de hospitalização por DM em ambos os sexos e todas as faixas etárias estudadas, com quedas mais expressivas no sexo feminino, apesar de evidenciarem os maiores coeficientes em todas as faixas etárias e período em estudo. Ainda, observou-se que, em idades mais avançadas, os coeficientes de hospitalização foram mais altos.

Tais resultados encontrados são relevantes pois, com a evidência de quedas nas taxas de hospitalização por DM, pode-se inferir que políticas públicas estão implementadas adequadamente, além de uma boa adesão ao tratamento por parte dos pacientes, e ainda, uma possível conscientização da população acerca da problemática que envolve o $\mathrm{DM}$, especialmente o público feminino.

Em contrapartida, a menor queda evidenciada no sexo masculino pode sinalizar a necessidade de implementar ações mais voltadas a esse público, de modo a conscientizar e obter a maior valorização da doença pelos homens.

O estudo apresenta algumas limitações relacionadas à utilização de dados secundários, passíveis de erros de codificação dos diagnósticos, além do fato de não ser possível identificar casos de reinternações e não ter sido considerada a mudança na disposição de leitos, bem como internações decorrentes de outras comorbidades, principalmente por indivíduos mais idosos, que sabidamente procuram o serviço de saúde por causas diversas.

Todavia, os resultados são de extrema importância pela possibilidade de indicar a relevância da implementação de ações no âmbito da assistência ambulatorial, que pode contribuir para limitar as complicações e preveni-las, evidenciando a necessidade de investimentos ainda maiores nessa direção de complexidade por parte dos gestores de saúde, além dos investimentos implantados até então.

São inúmeros os desafios na consolidação da Atenção Básica no país, e, sem dúvida, o aumento da cobertura é um passo fundamental. Frisa-se que ainda são necessários outros passos relativos para a melhoria da gestão, integração da atenção básica com a rede de serviços de saúde, financiamento, resolutividade, qualidade da atenção, entre outros, pois são fatores que juntos, irão trazer o que é essencial para a garantia do atendimento equânime e integral.

Ademais, reconhece-se a insipiência de estudos que investigam o comportamento dos coeficientes de hospitalização de uma das condições mais sensíveis à APS tal como o DM, de modo a fornecer um panorama indireto da qualidade de tal serviço. Para além disso, estudos desta natureza, promovem a reflexão de gestores e profissionais de saúde, acerca da efetividade das ações desenvolvidas junto às pessoas com $\mathrm{DM}$, de modo a promover melhorias nos quadros de pacientes atendidos pelo sistema público.

\section{REFERÊNCIAS}

1. Sociedade Brasileira de Diabetes. Diretrizes 2017 2018. São Paulo. Clannad, 2017.

2. World Health Organization. Global reporton diabetes [acessoemjun 2016]. Disponívelem: http://apps.who. int/iris/bitstream/10665/204871/1/9789241565257 eng.pdf?ua $=1$

3. International Diabetes Federation. Diabetes Atlas. 
International Diabetes Federation. $7^{\text {th }}$ Edition, 2015 [acesso em jun 2016]. Disponível em: http://www. diabetesatlas.org

4. Brasil. Ministério da Saúde. Vigilância de doenças crônicas não transmissíveis (DCNT). [acesso em set 2018]. Disponível em: http://portalms.saude.gov.br/ vigilancia-em-saude/vigilancia-de-doencas-cronicasnao-transmissiveis-dent.

5. Brasil. Ministério da Saúde. Departamento de Vigilância de Doenças e Agravos não Transmissíveis e Promoção da Saúde. Vigitel Brasil 2017: vigilância de fatores de risco e proteção para doenças crônicas por inquérito telefônico. - Brasília: Ministério da Saúde, 2018.

6. Cortez DN, Reis IA, Souza DA, Macedo MM, Torres HC. Complicações e o tempo de diagnóstico do diabetes mellitus na atenção primária. Acta Paul Enferm. 2015; 28 (3):250-5.

7. Barbosa VSN, Gomes LS, Palma DCA. Dislipidemia em pacientes com diabetes tipo 2. Rev. Saúde e Pesquisa. 2017; 10(3):579-85.

8. Arruda GO, Schmidt DB, Marcon SS. Internações por diabetes mellitus e a estratégia saúde da família, Paraná, Brasil, 2000 a 2012. Ciênc. Saúde coletiva. 2018; 23(2): 123-34.

9. Gibson OR, Segal L, Mcdermott RA. A systematic review of evidence on the association between hospitalization for chronic disease related ambulatory care sensitive conditions and primary health care resourcing. BMC Health Serv Res [Acesso em: 10 de maio de 2018]. 2013; 13: 336. Disponível em: https:// www.ncbi.nlm.nih.gov/pubmed/23972001

10. Brasil. Instituto Brasileiro de Geografia e Estatística - IBGE, 2016. Pesquisa Nacional por Amostra de Domicílios: síntese de indicadores 2016. Rio de Janeiro: IBGE.
11. Malta DC, Santos MAS, Stopa SR, Vieira JEB, Melo EA, Reis AAC. A Cobertura da Estratégia de Saúde da Família (ESF) no Brasil, segundo a Pesquisa Nacional de Saúde. Ciência e Saúde Coletiva [revista em internet]. 21(2):327-38. [Acesso em: 15 de maio de 2018]. Disponível em: http://dx.doi. org/10.1590/1413-81232015212.23602015.

12. Pinto HA, Sousa ANA, Ferla AA. O programa nacional de melhoria do acesso e da qualidade da atenção básica: várias faces de uma política inovadora. Revista Saúde Debate, 2014; 38(n. especial):358-72.

13. Malta DC, Merhy, EE. O percurso da linha do cuidado sob a perspectiva das doenças crônicas não transmissíveis. Interface: Comunicação, Saúde e Educação. 2010, 14(34):593-605.

14. Pereira DS, Devidé JÁ, Silva CAB. Qualidade de vida e situação de saúde dos idosos: um estudo de base populacional de base central o sertão do Ceara. Revista Bras Geriatria e Gerontologia. 2015, 18(4):893-908.

15. Clarec CS. Contribuição das internações por condições sensíveis à atenção primária no perfil das admissões pelo sistema público de saúde. Revista Pan-americana de saúde pública. 2013; 34(4):22734.

16. Flor LS, Campos MR. Prevalência de diabetes mellitus e fatores associados na população adulta brasileira: evidências de um inquérito de base populacional. Revista Brasileira de Epidemiologia [revista em internet]. 2017 [Acesso em 02 setembro 2018] 20(01);16-29. Disponível em: https://doi. org/10.1590/1980-5497201700010002.

17. Santos RR, Schmidt MI, Duncan BB, Souza MFM, Lima AK, Moura L. Internações por Diabetes Mellitus como diagnóstico principal na Rede Pública do Brasil. Revista. Brasileira de Epidemiologia. 2007, $10(4) ; 465-78$. 
18. Levorato $C D$. Fatores associadosà procura por serviços de saúde: diferenças entre mulheres e homens [dissertação]. Ribeirão Preto: Universidade de São Paulo, Faculdade de Medicina de Ribeirão Preto, 2012, [citado 2018-09-05]. doi:10.11606/D.17.2012. tde-21112012-093150.

19. Nunes AA, Levorato CD, Mello LM, Silva AS. Fatores associados à procura por serviços de saúde numa perspectiva relacional de gênero. Cien Saude Colet [periódico na internet]. 2014 [Citado em 20/10/2018]; 19(4):1263-74. Disponível em: http://www.cienciaesaudecoletiva. com.br/artigos/fatores-associados-a-procura-porservicos-de-saude-numa-perspectiva-relacional-degenero/12756 id=12756\&id=12756.

20. Santos AL, Teston EF, Latorre MRDO, Mathias TAF, Marcon SS. Tendência de hospitalizações por diabetes mellitus: implicações para o cuidado em saúde. Acta Paul Enferm. 2014. [Acesso em: 15 de maio de 2018]. 2015; 28(5):401-7. Disponível em: http://www.scielo. br/pdf/ape/v28n5/1982-0194-ape-28-05-0401.pdf.

21. Tong SF, Low WY, Ismail SB, Trevena L, Willcock S. Malaysian primary care doctors' views on men's health: an unresolved jigsaw puzzle. BMC Fam Pract [Acesso em: 12 de fev de 2018]. 2011. 12:29. Disponível em: https://doi.org/10.1186/14712296-12-29.

22. MaltaDC, Bernal RTI, LimaMG, Araújo SSC, Silva MMA, Freitas MIF, et al. Doenças crônicas não transmissíveis e a utilização de serviços de saúde: análise da Pesquisa Nacional de Saúde no Brasil. Revista Saúde Pública [Acesso em: 02 de mar de 2018]. 2017; 51 Supl 1:4. Disponível em: http://www.scielo.br/scielo. php?pid $=$ S0034-89102017000200306\&script $=$ sci arttext\&tlng $=\mathrm{pt}$

23. Sousa, JCO, Carvalho CR. Resistência Masculina pela Atenção à Saúde. Rev Científ Multidisciplinar Núcleo do Conhecimento. 2017, 07(09); 5-16. 